\title{
NS1 of H7N9 Influenza A Virus Induces NO-Mediated Cellular Senescence in Neuro2a Cells
}

\author{
Yinxia Yan ${ }^{\mathrm{a}}$ Yongming Du ${ }^{\mathrm{b}}$ Huali Zheng ${ }^{\mathrm{a}}$ Gefei Wang ${ }^{\mathrm{a}}$ Rui Li ${ }^{\mathrm{a}} \quad$ Jieling Chen ${ }^{\mathrm{c}}$ \\ Kangsheng Li ${ }^{\mathrm{a}}$ \\ aKey Laboratory of Infectious Diseases and Molecular Immunopathology of Guangdong Province, \\ Department of Microbiology and Immunology, Shantou University Medical College, Shantou, \\ Guangdong Province, China, 'Department of Biochemistry, the University of Hong Kong, Hong Kong, \\ 'Shantou-Oxford Clinical Research Unit, Shantou University Medical College, Shantou, Guangdong \\ Province, China
}

\section{Key Words}

H7N9 influenza virus $•$ NS1 • Nitric oxide $•$ Cellular senescence $•$ p38 MAPK

\begin{abstract}
Background/Aims: The novel avian H7N9 influenza A virus has been detected in brain tissues and associated with central nervous system (CNS) symptoms in infected human and mice. Roles of its virulence factor, NS1 protein in influenza virus infected neuron has yet to be explored. Methods: Nitric oxide (NO) release and inducible nitric oxide synthase (iNOS) expression in H7N9/NS1-expressed Neuro2a cells were detected by Griess test and western blotting. Cell proliferation rate of H7N9/NS1-expressing cells was recorded by Cell Counting Kit-8. Effects of H7N9/NS1 on cellular senescence were investigated by senescenceassociated $\beta$-galactosidase (SA- $\beta$-gal) staining, immunofluorescent staining of phosphorylated heterochromatin protein $1 \gamma$ (pHP1 $\gamma$ ) and qPCR analysis of IL- 6 and IL-8. Results: H7N9/NS1 in Neuro2a cells and primary cultured mouse cortical neurons increased the expression of iNOS and boosted NO release. Neuro2a cells constitutively expressing NS1 displayed a reduced proliferative ability, enhanced SA- $\beta$-gal staining, increased level of IL- 6 and IL- 8 and a typical punctuate structure of pHP1 $\gamma$ in nuclei. In addition, p38 MAPK was elevated in NS1-expressing Neuro2a cells. Reduced iNOS expression and subdued cellular senescence effect was found in p38 MAPK inhibitor-treated NS1-expressing Neuro2a cells. Conclusion: Our results suggest that H7N9/NS1 protein increases the iNOS expression and NO release in Neuro2a cells, which can induce cell growth arrest and cellular senescence.
\end{abstract}




\section{Cellular Physiology Cell Physiol Biochem 2017;43:1369-1380

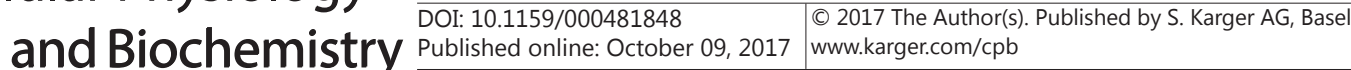 \\ Yan et al.: H7N9 NS1 Protein Induces Cellular Senescence}

\section{Introduction}

The high mortality rates associated with H7N9 influenza A virus infection in humans remains a serious health concern as well as a significant economic burden. Since the first cases of human avian influenza A (H7N9) in China were reported to the World Health Organization, over 1260 infections in humans have been reported, of which approximately $40 \%$ have been fatal [1]. Although most H7N9 virus infections in patients primarily affect the respiratory system, leading to respiratory failure and acute respiratory distress syndrome, central nervous system (CNS) symptoms have been associated with the novel H7N9 influenza $A$ virus [2]. Previous studies have shown that several strains/subtypes of influenza A virus, such as $2009 \mathrm{H} 1 \mathrm{~N} 1$ pandemic virus [3, 4], HPAI H5N1 virus [2, 5, 6] and H7N7 virus [7, 8] have been associated with the development of CNS diseases in human, ferret or murine models, which is considered as the most common extra-respiratory complication of influenza [9].

Neuron is thought to be the predominant infected cell type by influenza A virus in the CNS in ferret and murine models [10]. Infection of human influenza A virus in mouse neurons upregulates cytokines, chemokines and type I interferons, leading to neuron injury to some extent [11]. Accumulating evidence suggests that the inflammatory activation of neurons may serve as a source of cytotoxic agents and reactive oxygen species in brain [12]. Reactive oxygen species may directly contribute to cell death and exacerbate pathology caused by virus replication during influenza virus infection $[13,14]$. As one of many freeradical molecules produced by inflammatory cells during influenza virus infection, nitric oxide (NO) has been suggested to contribute to the disease process [15-17]. The production of NO in brain is catalyzed by 3 different isoforms of the nitric oxide synthase (NOS). NO derived from the constitutive, calcium-dependent isoforms nNOS and eNOS is involved in neural signaling and vasodilation [18]. Overproduction of NO is mainly caused by inducible NO synthase (iNOS), which is usually expressed by inflammatory phagocytic cells and other types, such as epithelial and neuronal cells [19]. In brain, iNOS expression has been well characterized in astrocytes, microglia cells and endothelial cells. Glia iNOS expression has been described after injury or trauma, and has been implicated in several pathologies [20]. Ongoing expression of iNOS in neurons has for many years been associated with deleterious consequences. Induction of iNOS by cytokines has been shown to induce cell death in both PC12 cells and primary cerebellar granule neurons [21, 22]. Competitive NOS inhibitors can rescue neurons from death through the inhibition of iNOS activity [23]. NO derived from iNOS is often involved in cytotoxic and cytostatic functions, contributing to the destruction of invading organism and tumor cells [18], as well as cellular senescence [24].

iNOS is induced in a variety of experimental virus infections in rats and mice, including those with influenza virus $[15,19,25]$. The multifunctional NS1 protein of influenza A virus is widely regarded as a virulence factor, and contributes significantly to disease pathogenesis by modulating many viral and cellular processes [26]. A recent study suggested NS1 is one of the most important factor in the induction of iNOS promoter activities in human cells [27]. Our previous study has shown that NS1 protein of H7N9 virus induces p53-mediated apoptosis of human A549 cells [28]. This study was initially aimed to examine whether H7N9/ NS1 induced apoptosis in Neuro2a cells, a mouse neuroblastoma cell line. However, the data detailed below led to an unexpected link between H7N9/NS1 and cellular senescence in Neuro2a cells, suggesting a novel role for NS1 protein in influenza virus-infected neuron cells.

\section{Materials and Methods+}

Cell culture

Mouse Neuroblastoma Neuro2a Cells and Human Embryonic Kidney 293T Cells obtained from the Cell Bank of the Chinese Academy of Sciences (Shanghai, China) were cultured at $37^{\circ} \mathrm{C}$ with $5 \%$ CO2 in DMEM/ F12 and DMEM supplemented with $10 \%$ FBS, respectively. 


\section{Cellular Physiology Cell Physiol Biochem 2017;43:1369-1380 and Biochemistry Published $\begin{aligned} & \text { DOI: 10.1159/000481848 } 2017 \text { The Author(s). Published by S. Karger AG, Basel } \\ & \text { www.karger.com/cpb }\end{aligned}$}

Yan et al.: H7N9 NS1 Protein Induces Cellular Senescence

Animal study was preapproved by Ethical Committee of Shantou University Medical College. Pregnant C57BL/6J mice were purchased from Shantou University Medical College Laboratory Animal Center, Shantou, Guangdong. Primary cortical neuron cells from neonatal mouse were isolated and cultured in neurobasal medium supplemented with $2 \%$ B27, 2 mM L-Glutamine and 0.5\% penicillin-streptomycin at $37^{\circ} \mathrm{C}$ in $5 \% \mathrm{CO} 2$.

\section{Transient transfection}

H7N9/NS1 cDNA was cloned into pcDNA3-Flag vector (a modified pcDNA3 vector providing an $\mathrm{N}$-terminal Flag tag) as described previously [28]. Neuro2a cells were inoclulated into 6-well plate $\left(2 \times 10^{6} /\right.$ well) and transfected with $3 \mu \mathrm{g}$ plasmid DNA using Lipofectamin 2000 (Life Technology, USA). Control cells were transfected with an equal amount of the parental vector alone. Transfected cells were then harvested $0,6,12,24,36$ and $48 \mathrm{~h}$ post-transfection.

\section{NS1-lentivirus construction, packaging and infection}

The lentivirus packaging system (Origene Technologies, USA) was used to construct and package H7N9/ NS1-lentivirus according to the Manufacturer's protocol. Briefly, H7N9/NS1 cDNA was cloned into pLentiFlag-Myc vector using EcoR I and Nhe I restriction endonucleases. The verified construct was co-transfected HEK293T cells with pCMV-dR8.91 and pMD2G-VSVG using lipofectamine 2000. Medium containing recombinant lentivectors at $48 \mathrm{~h}$ post-transfection was harvested and centrifugated. Supernatant was filtrated through $0.45 \mu \mathrm{m}$ filter unit, aliquoted and stored at $-80^{\circ} \mathrm{C}$. Primary cultured mouse cortical neurons were infected by NS1-lentivirus or lentiviral control (mock-lentivirus) at an MOI of 5 for $72 \mathrm{~h}$.

Caspase-Glo 3/7 Assay, Trypan blue assay and LDH assay

For cytotoxic and apoptosis assay of H7N9/NS1-expressed Neuro2a cells, we used protocols previously described [28]. Each experiment was repeated at least three times.

\section{Stable cell line generation}

Construct encoding H7N9/NS1 above-mentioned was digested with Kpn I and EcoR I restriction endonucleases (New England Biolabs, Germany) and ligated into pcDNA3-Flag/Puro vector (a modified pcDNA3 vector providing an N-terminal Flag tag and a puromycin selection marker). The verified construct was transfected in Neuro2a cells to generate H7N9/NS1 expressing stable cell lines. Puromycin $(1 \mu \mathrm{g} / \mathrm{ml})$ was added $36 \mathrm{~h}$ post-transfection to select the transfected cells and $10 \mathrm{mM}$ S-methylisothiourea sulfate (SMT, Sigma) was added 3 days later to avoid the toxicity of intracellular NO. Single cell clones were selected into 24-well plate after about 2 weeks. The positive cell clones were confirmed by verifying expression of H7N9/NS1 protein by western blotting.

RNA preparation, RT-PCR, and real-time Quantitative PCR ( $q$ PCR)

Total RNA was extracted from transfected Neuro2a cells and lentivirus-infected cortical neurons using TRIzol reagent (Invitrogen). RNA $(1 \mu \mathrm{g})$ from each sample was reverse-transcribed into cDNA using the PrimeScript ${ }^{\circledR}$ RT reagent kit (Takara). qPCR was carried out in an Eco Real-Time PCR System (Illumina, USA) using ChamQ ${ }^{\mathrm{TM}} \mathrm{SYBR}^{\circledR}$ qPCR Master Mix (Vazyme, China). qPCR primers used are as follows: iNOS primers (forward, 5'-TCCACAG TATGTGAGGATCAAAAAC-3' and reverse, 5'- TCCACAGTATGTGAGGATCAAAAAC-3'), eNOS primers (forward, 5'-GATGGGCCCTGTACCT CAA-3' and reverse, 5' - GTGGGCCGGCTCTGTAA CT-3'), nNOS primers (forward, 5'AAAACCTG CAAAGTCCTAAATCCA-3' and reverse, 5'- CTCC TGATTCCCGTTGGTGT-3'), IL-6 primers (forward, 5'- CCAGTTGCCTTCTTGGGACT-3' and reverse, 5'-GGTCTGTTGGGAGTGGTATCC-3'), IL-8 primers (forward, 5'- GCATGAAAAGCCT CTATA-3' and reverse, 5'- AAATCAGCAAAGTGT CTA-3') and GAPDH primers (forward, 5'- GCATTGTGGAAGGGCTCA-3' and reverse, 5'- AGGCGGCACGTCAGATC-3').

\section{Detection of NO}

The supernatants of transfected Neuro2a cells and lentivirus-infected mouse cortical neurons were collected at various time points as indicated, and immediately used to perform Griess test using Total NO Assay Kit (Beyotime, China) according to the Manufacturer's protocol. Equal amount of reagents and sample were mixed and added to 96 -well plate and incubated for $30 \mathrm{~min}$ at $37^{\circ} \mathrm{C}$. The absorbance was then read at $540 \mathrm{~nm}$ and the amount of nitrate were calculated using the standard curve. 


\section{Cellular Physiology Cell Physiol Biochem 2017;43:1369-1380 \begin{tabular}{l|l|l} 
and Biochemistry Published online: October 09, 2017 & $\begin{array}{l}\text { (c) } 2017 \text { The Author(s). Published by S. Karger AG, Basel } \\
\text { www.karger.com/cpb }\end{array}$ \\
\hline
\end{tabular}}

Yan et al.: H7N9 NS1 Protein Induces Cellular Senescence

\section{Western blot analysis}

Western blot analysis was performed by standard method using the following antibodies: anti-Flag (Sigma; cat no: F1804) for Flag-tagged NS1 protein, anti-PARP (Cell Signaling Technology; cat no: 9542) for Poly (ADP ribose) polymerase, anti-iNOS (Proteintech; cat no: 18985-1-AP) for iNOS, monoclonal rabbit p38 MAPK (D13E1) antibody (Cell Signaling Technology; cat no: 9212) for total p38 MAPK, monoclonal rabbit phospho-p38 MAPK (Thr180/Tyr182) antibody (Cell Signaling Technology; cat no: 9211) for phosphorylated p38 MAPK and anti- $\beta$-actin monoclonal antibody (Sigma; cat no: A5441) was used as the loading control. Each experiment was repeated at least three times.

\section{Cell proliferation assay}

A cell counting kit (CCK-8) assay (Life technology) was used to measure cell proliferation according to the manufacturer's guidelines. Briefly, cells were seeded in 96-well microtiter plates (1, 000 cells per well) and incubated with DMEM/F12 containing 10\% FBS. After incubation for the indicated days, $10 \mu \mathrm{l}$ CCK8 was added into each well, followed by continuous incubation for 4 h. The optical density value of each sample was measured at a wavelength of $450 \mathrm{~nm}$ on a microplate reader. Each experiment was carried out in triplicate.

Senescence Associated $\beta$ Galactosidase (SA- $\beta$-gal) Assay

Senescence $\beta$-Galactosidase Staining Kit (Beyotime, China) was used for determining senescence in H7N9/NS1 stable expressing Neuro2a cells according to the manufacturer's instructions. The cells were imaged using 20x objective on an inverted IX81 microscope with DP71 color camera (Olympus, Japan) for presence of the blue color staining.

\section{Immunofluorescence microscopy}

Neuro2a cells stably expressed H7N9/NS1 were seeded onto coverslips in 24-well plate. Cells were washed with PBS and fixed with 4\% paraformaldehyde solution for 5 min and permeabilized in $0.2 \%$ Triton X-100, $0.04 \%$ SDS for 3 min. After blocking in 10\% normal goat serum (Boster, Wuhan, China) at room temperature, cells were incubated overnight at $4{ }^{\circ} \mathrm{C}$ with primary antibody against phosphorylated heterochromatin protein $1 \gamma$ (pHP1 $\gamma$ ) (Cell Signaling Technology; cat no: 2600). The appropriate Alexa-Fluorcoupled secondary antibody (Invitrogen; 1:750) was applied for $1 \mathrm{~h}$ at room temperature. The cells were mounted in ProLong Gold antifade reagent with 6-diamidino-2-phenylindole (DAPI) (Life Technologies) and analyzed using Nikon Eclipse 80i microscope (Nikon, Japan).

\section{Results}

H7N9/NS1 induces Neuro2a cell death independent of apoptosis

To assess the expression of NS1 protein in Neuro2a cells after transfection with the pcDNA3-Flag-NS1 plasmid, cells were collected $0,6,12,24,36$, and $48 \mathrm{~h}$ post-transfection. The NS1 protein expression was detectable as early as $12 \mathrm{~h}$ and peaked by $36 \mathrm{~h}$ post-transfection (Fig. 1A). Trypan blue dye exclusion assay and LDH assay were done to detect the cell viability and lysis following NS1 expression at different intervals. A decreased percentage of live cells and increased percentage of cell lysis were found in NS1-transfected Neuro2a cells, compared to the mock-transfected cells (Fig. 1B and C). Caspase 3/7 activity assay and western blot analysis of PARP cleavage were performed to examine whether H7N9/NS1 was able to modulate apoptosis in Neuro2a cells, just as in human A549 cells. However, no significant activation of caspase 3/7 (Fig. 1D) and cleavage of PARP (Fig. 1E) was detected in both mock-transfected and NS1-transfected Neuro2a cells at $36 \mathrm{~h}$ post-transfection. These data showed that H7N9/NS1 induces cell death independent of apoptosis in Neuro2a cells.

Expression of H7N9/NS1 boosted NO release and increased iNOS expression in Neuro2a cells and mouse cortical neurons

Our previous study revealed that NS1 of H5N1 influenza virus significantly reduces the production of NO in rat hippocampal neurons [29], while NS1 of H1N1 was the opposite 


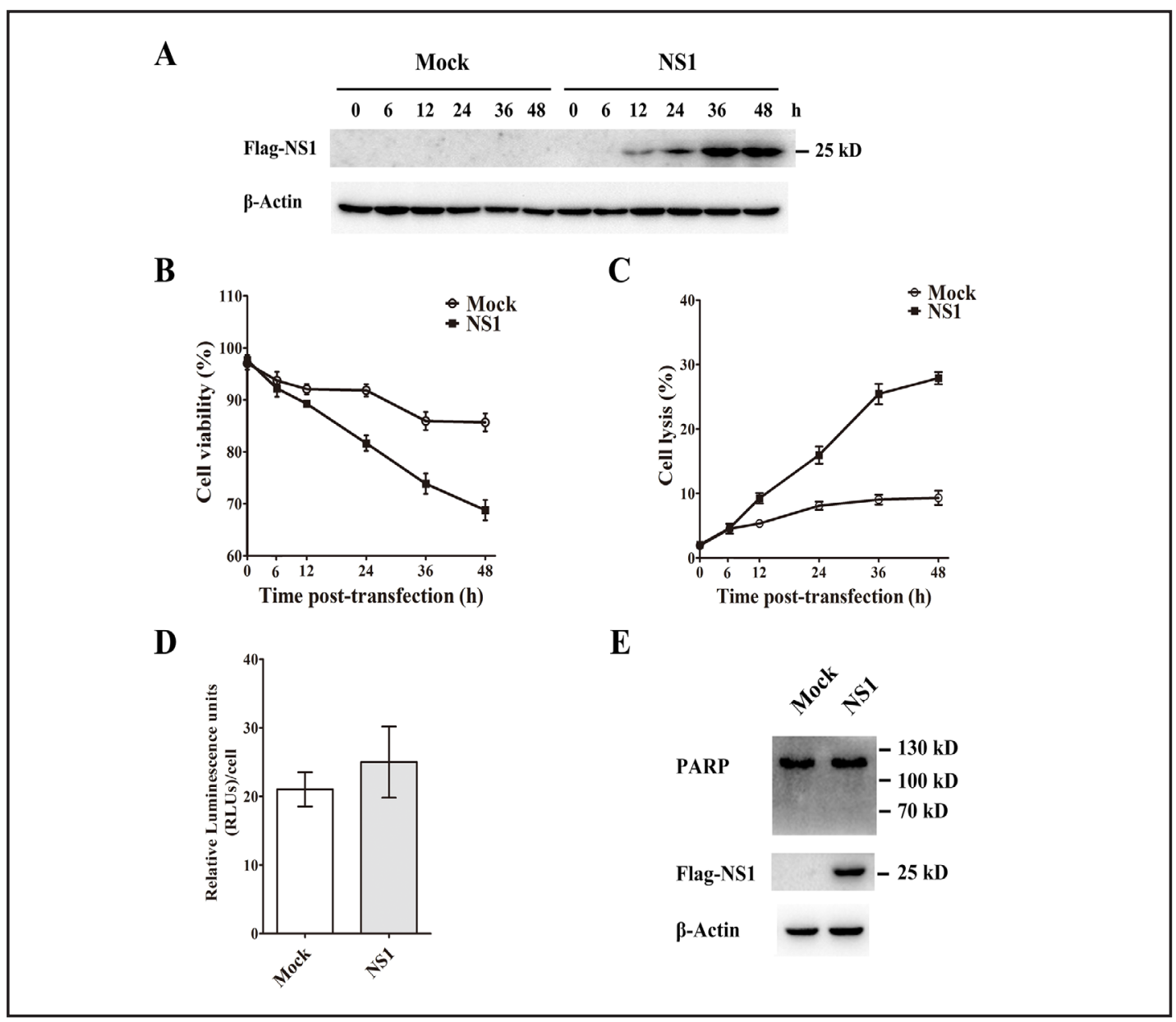

Fig. 1. H7N9/NS1 induces Neuro2a cell death independent of apoptosis. Transfected cells at different time intervals $(0,6,12,24,36$ and $48 \mathrm{~h})$ were harvested and processed for western blot analysis (A), trypan blue assay (B) and LDH assay (C). Cells at $36 \mathrm{~h}$ post-transfection were harvested and subjected to caspase 3/7 activity assay of apoptosis (D) and Western blot analysis of PARP cleavage (E). Mock, cells transfected with pcDNA3-Flag empty vector; NS1, cells transfected with pcDNA3-Flag-H7N9/NS1.

(data not shown). Given NS1 from different influenza A virus strains exhibited distinct properties in promoting NO release in host cell, it was initially tested if H7N9/NS1 can promote production of nitric oxide in Neuro2a cells and primary cultured cortical neurons. As shown in Fig. 2A (Left panel), rapid release of NO was detectable after $12 \mathrm{~h}$ post-transfection, and NO level was increased with the increase expression of NS1 protein in H7N9/NS1expression Neuro2a cells. It can be seen in Fig. 2A (Right panel) that the production of NO was elevated in NS1-lentivirus infected mouse cortical neurons compared with mock-lentivirus infected and untreated neurons. Given that the level of NO was significantly increased with expression of NS1 in Neuro2a cells and neurons, it was important to establish that iNOS was increased accompany with the increased NS1 expression. We evaluated mRNA level of NOSs in transfected Neuro2a cells at $36 \mathrm{~h}$ post-transfection (Fig. 2B, Left panel). This time point was selected based on the observation that NS1 was highly expressed and NO was rapidly released $36 \mathrm{~h}$ post-transfection. Results of qPCR revealed that mRNA level of iNOS in H7N9/ NS1-transfected Neuro2a cells increased by about 17.5 -fold compared with the mock, while no significant change in the mRNA level of nNOS and eNOS can be observed (Fig. 2B, Left panel). In addition, mRNA and protein levels of iNOS in NS1-lentivirus infected neurons were also highly elevated compared with mock-infected and untreated neurons (Fig. 2B, Right panel and Fig. 2C, Right panel). Additional, increase of the protein level of iNOS was in a 
time dependent manner to some extent (Fig. 2C, Left panel). Collectively, all these results indicated that H7N9/NS1 boosted NO release and increased iNOS expression in Neuro2a cells and primary cultured mouse cortical neurons.

\section{H7N9/NS1 inhibits Neuro2a cell proliferation}

Given that the presence of excessive NO can cause cell growth arrest, we next take advantage of Neuro2a cells stably expressing H7N9/NS1 to examine the effect of H7N9/ NS1 on cell proliferation. Towards this, H7N9/NS1 was stably transfected to Neuro2a cells and after about 4 days, expression of iNOS increased remarkedly, and significant cell death was found indicating that the H7N9/NS1 was lethal (data not shown). Given that excessive expression of iNOS is cytotoxic, 10 mM SMT working as iNOS specific inhibitor, was added into cell culture medium to overcome the iNOS cytotoxicity and rescue cells. Neuro2a cell with H7N9/NS1 stably expressed (NS1 O.E) were kept in presence of SMT (10 mM) for 2 days, and then were split to 2 sets on the next day. One set, the cells were maintained in medium containing $10 \mathrm{mM}$ SMT (NS1 0.E (+SMT)), and in the other set, SMT was removed (NS1 O.E (-SMT)). Expression of H7N9/NS1 and iNOS were confirmed by western blot analysis on day 3 (Fig. 3A) and concomitantly increase in NO levels of NS1 O.E (-SMT) compared with NS1 O.E (+SMT) and normal Neuro2a cells (WT) were recorded by Griess test (Fig. 3B). Notably, NS1 O.E cells which maintained without SMT looked morphologically similar to senescent cells at day 3, and exhibited excessive cell death at day 7 (Fig. 3C, right

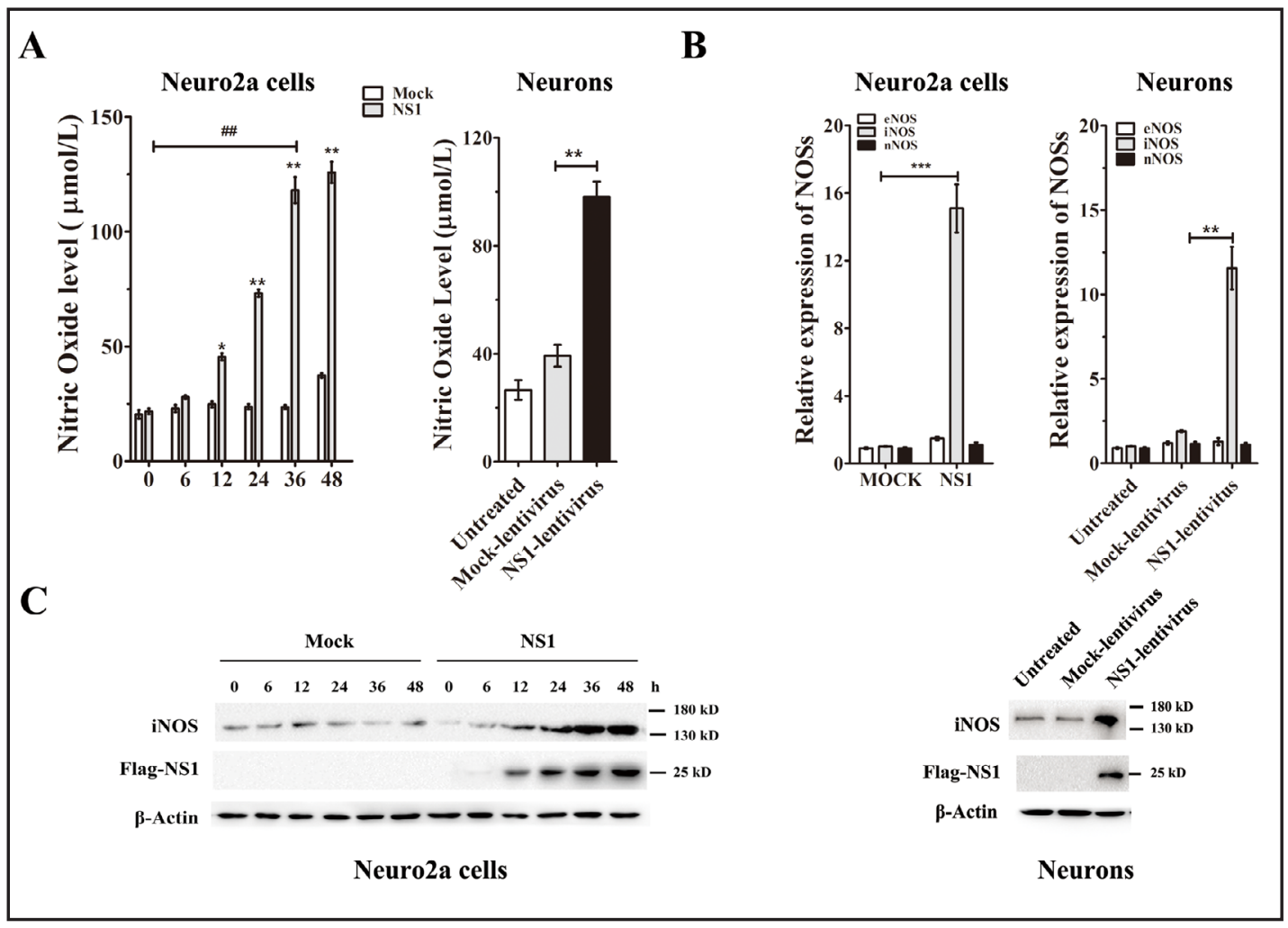

Fig. 2. H7N9/NS1 boosts NO release and increases iNOS expression in Neuro2a cells and mouse cortical neurons. (A) No release dynamics in H7N9/NS1-expressing Neuro2a cells (Left panel) and mouse cortical neurons (Right panel). *, p<0.05 (t test); **, p<0.01 ( $\mathrm{t}$ test); \#\#, p<0.01 (t test). (B). mRNA levels of eNOS, iNOS, and nNOS in H7N9/NS1 transfected Neuro2a cells (Left panel) and mouse cortical neurons (Right panel) were detected by qPCR. Results show the mean of three expriments \pm s.e.m and are normalized to the GAPDH gene. ${ }^{* *}, \mathrm{p}<0.01$ ( $\mathrm{t}$ test). (C) Western blot analysis of iNOS and Flag-NS1 in transfected neuro2a cells (Left panel) and lentivirus-infected neurons (Right panel). $\beta$-actin was used as loading control. 
panels), while NS1 O.E (+SMT) cells showed normal appearance of Neuro2a cells at day 3, but a decreased proliferative ability at day 7 (Fig. 3C, middle panels). Cell proliferation was compared between Neuro2a cells (WT) and NS1 O.E (+SMT) cells. As shown in Fig. 3D, cells were cultured for 4, 5, 6 and 7 days, the proliferation of NS1 O.E (+SMT) exhibited a slight downward trend, and significantly lower than the proliferation of the WT cells on day 5, day 6 and day 7 ( $\mathrm{p}<0.01)$, indicating that expression of H7N9/NS1 inhibited the proliferation of Neuro2a cells to some extent.

\section{H7N9/NS1 induces cellular senescence in Neuro2a cells}

As mentioned above, NS1 O.E (-SMT) cells showed a senescent cells-like morphology on day 3, and stained positive for SA- $\beta$-Gal (Fig. 4A, upper right panel), compared with the NS1 O.E (+SMT) or WT cells. Senescent cells often show widespread chromatin modifications, such as phosphorylated heterochromatin protein $1 \gamma$ (pHP1 $\gamma$ ), HMGA1 or $\gamma \mathrm{H} 2 \mathrm{~A}$ [30]. Next, we examined the structure of pHP1 $\gamma$ in NS1 O.E (-SMT) cells using immunofluorescence. Fluorescence microscopy of these cells revealed typical punctuate structures of in the nuclei (Fig. 4A, low right panel). Cytokines IL-6 and IL-8 have been shown to play a critical role in cellular senescence [31]. We next examined the mRNA level of inflammatory cytokines, IL-6 and IL-8 by qPCR. As shown in Fig. 4B, both IL-6 and IL-8 were significantly enhanced in H7N9/NS1 stably expression Neuro2a cells, compared with NS1 O.E (+SMT) and WT cells. Overall, it was determined that expression of H7N9/NS1 induces senescence in Neuro2a cells, which might be associated with the high expression of iNOS.

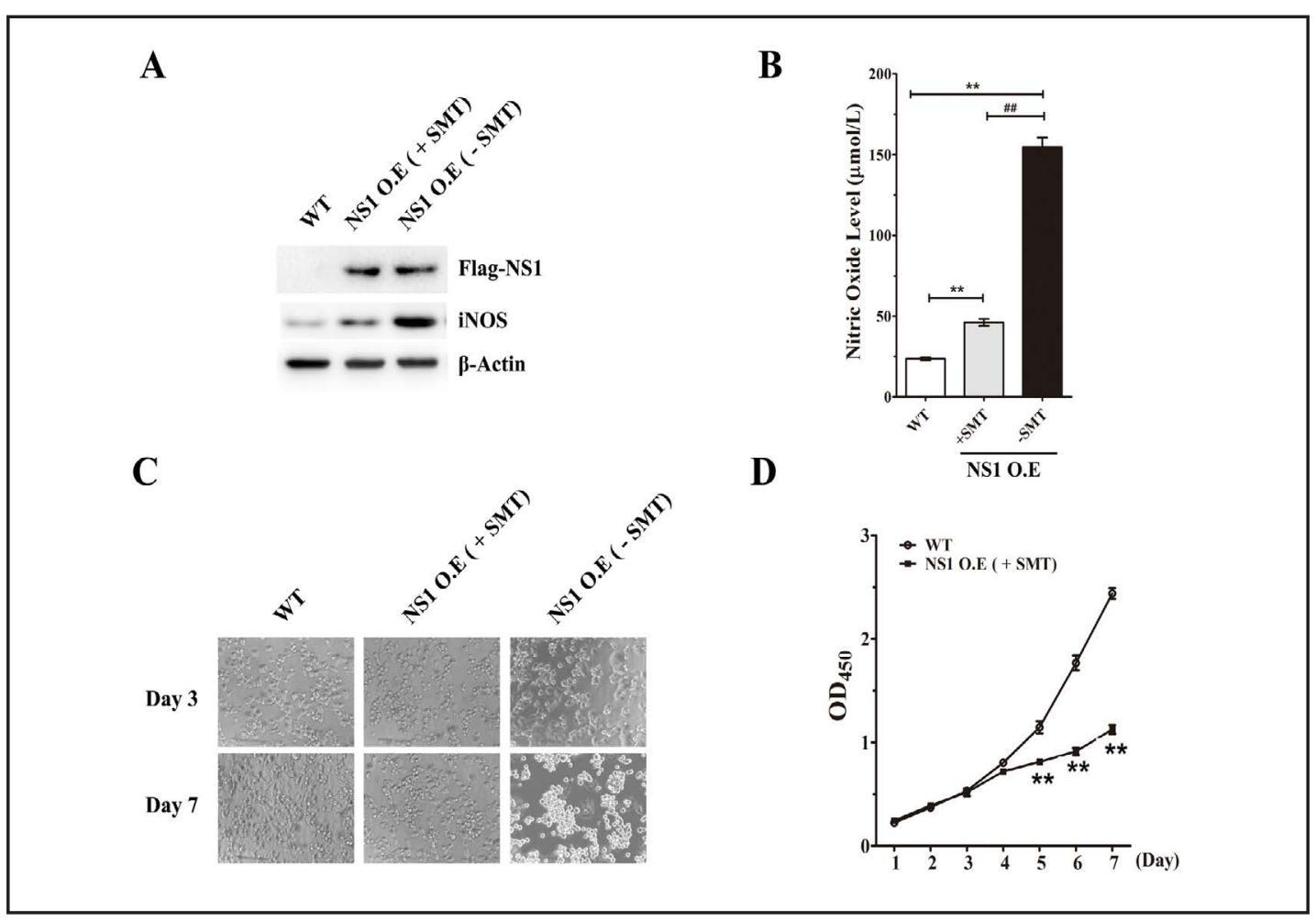

Fig. 3. H7N9/NS1 inhibits Neuro2a cell proliferation. H7N9/NS1 expression plasmid with a puromycin selection marker was transfected and the cells were grown in presence of $10 \mathrm{mM}$ SMT and $1 \mu \mathrm{g} / \mathrm{ml}$ puromycin. After 2 days, the cells were divided into two groups, with $10 \mathrm{mM} \mathrm{SMT}(\mathrm{NS} 1$ O.E (+SMT)) and without SMT (NS1 O.E (-SMT)). The Neuro2a cells without any treatment was marked as wild type (WT). (A) NS1 and iNOS expression confirmed by western blotting on day 3. (B) NO level quantitated by Griess reagent on day 3 (the third day after grouped). ${ }^{* *}, \mathrm{p}<0.01$ (t test); \#\#, $\mathrm{p}<0.01$ (t test). (C) Morphological changes in H7N9/NS1 stably expressing Neuro2a cells at day 3 and day 7. (D) Cell proliferation assay using cck-8. The number of viable cells was evaluated from day 1 to day 7 by cck-8. 
A

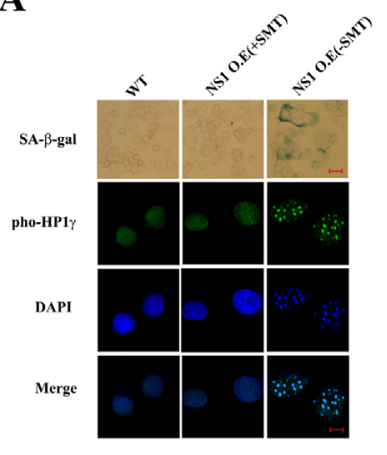

B

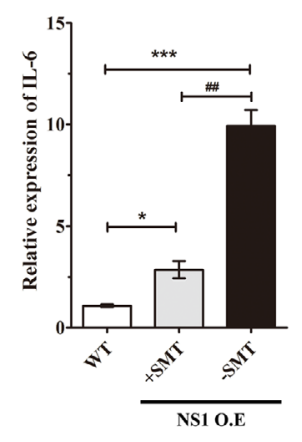

C

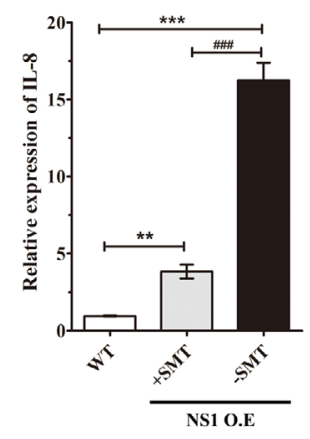

Fig. 4. H7N9/NS1 induces cellular senescence in Neuro2a cells. Neuro2a WT, NS1 O.E (+SMT) and NS1 O.E (-SMT) cells were grown in indicated medium for 2 days, then various markers were analyzed on day 3. (A) NS1 O.E (-SMT) cells showed enhanced $\beta$-alactosidase activity (upper panels), pHP1 $\gamma$-positive nuclear foci (lower panels) when compared with NS1 O.E (+SMT) and untreated WT cells. qPCR analysis reveals enhanced expression of cytokines IL-6 (B) and IL-8 (C) in NS1 O.E (-SMT) cells. Results of qPCR analyses are mean of three experiments \pm s.e.m. from three independent experiments. Scale bars: $75 \mu \mathrm{m}$ (SA- $\beta$-gal staining); $7.5 \mu \mathrm{m}$ (immunofluorescence). ${ }^{*}, \mathrm{p}<0.05$ versus WT (t-test); ${ }^{* *}, \mathrm{p}<0.01$ versus WT (t-test); ${ }^{* * *}$, $\mathrm{p}<0.001$ (t-test). \#\#\#, $\mathrm{p}<0.001$ verus NS1 O.E (+SMT) (t-test).

A

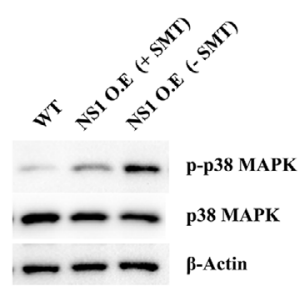

C

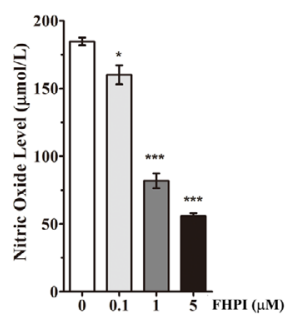

B

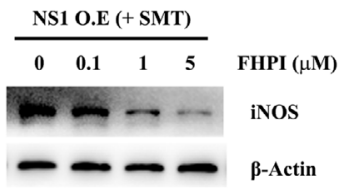

$\mathbf{E}$

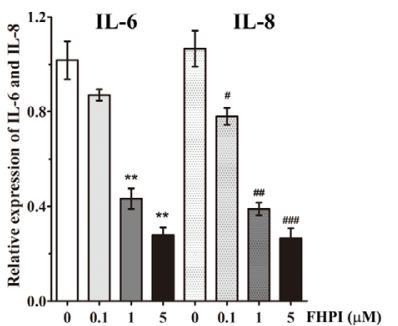

Fig. 5. p38 MAPK is involved in H7N9/NS1 induced cellular senescence in Neuro2a cells. (A) p38 MAPK was activated in NS1 O.E cells, especially NS1 O.E without SMT treatment cells. (B) NS1 O.E (-SMT) cells were cultured in indicated medium for 2 days, then were treated with various concentrations of p38 MAPK inhibitor, FHPI for $12 \mathrm{~h}$. iNOS expression was inhibited in a dose-dependent way, congruent with the increasing of FHPI concentration. The NO production in NS1 O.E (-SMT) Neuro2a cells cultured in the presence of FHPI agreed with the iNOS expression (C). (D) SA- $\beta$-gal assay of senescent cells grown in the presence of different concentration of FHPI. Percentage of SA- $\beta$-gal positive cells were calculated to measure proportion of senescent cells $(n=3) . * * *, p<0.001$ verus FHPI $(0 \mu M)$ treated cells (t-test) (E) qPCR analysis of IL- 6 and IL-8 expression in NS1 O.E (-SMT) Neuro2a cells with FHPI treatment. Results represent the mean of three independent experiments \pm s.e.m. * ${ }^{*}<<0.05$; **, p<0.01 (t-test); \#, $\mathrm{p}<0.05$; \#\#, p<0.01;\#\#\#, $\mathrm{p}<0.001$ (t-test).

\section{KARGER}




\section{Cellular Physiology Cell Physiol Biochem 2017;43:1369-1380 \begin{tabular}{ll|l} 
and Biochemistry & $\begin{array}{l}\text { DOI: 10.1159/000481848 } \\
\text { Published online: October 09, } 2017\end{array}$ & $\begin{array}{l}\text { O 2017 The Author(s). Published by S. Karger AG, Basel } \\
\text { www.karger.com/cpb }\end{array}$ \\
\cline { 1 - 2 }
\end{tabular}}

Yan et al.: H7N9 NS1 Protein Induces Cellular Senescence

p38 MAPK is involved in H7N9/NS1 induced cellular senescence in Neuro2a cells

It has been reported previously that p38 MAPK plays a critical role in NO-mediated cell death in human SH-SY5Y neuroblastoma cells and primary cultures of cortical neurons [32]. Blockage of p38 MAPK pathway will inhibit the expression of iNOS in Mouse Astrocytes [33]. We next examined the activation of p38 MAPK in the NS1 O.E cells. Activation was detected in NS1 O.E cells compared with WT cells by western blot analysis of total p38 MAPK and phosphorylated p38 MAPK. Particularly, NS1 O.E (-SMT) cells showed a more significant activation of p38 MAPK than NS1 O.E (+SMT), indicating that p38 MAPK activation is linked to the increase of expression of iNOS (Fig. 5A). To confirm the activation of p38 MAPK was involved in H7N9/NS1 induced cellular senescence, a specific inhibitor, FHPI (SB202190), was used to block p38 MAPK activity. As shown in Fig. 5B, preincubation of NS1 O.E (-SMT) cells with $1 \mu \mathrm{M}$ and $5 \mu \mathrm{M}$ FHPI substantially reduced the iNOS protein after $12 \mathrm{~h}$. NO level in cells culture in the presence of increasing concentrations of FHPI agreed well with iNOS expression (Fig. 5C). Percentage of cells staining positive for SA- $\beta$-Gal was significantly decreased in $1 \mu \mathrm{M}(28.9 \pm 2.01)$ and $5 \mu \mathrm{M}(16.0 \pm 2.83)$ FHPI pretreated cells, compared with FHPI-untreated cells $(85.1 \pm 2.95)$ (Fig. 5D). Furthermore, higher concentrations of FHPI $(1 \mu \mathrm{M}$ and $5 \mu \mathrm{M})$ pretreated cells resulted in a strong reduced IL- 6 and IL-8 expression compared to FHPI- untreated NS1 O.E (-SMT) cells (Fig. 5E). Taken together, these data suggest activation of p38 MAPK is involved in H7N9/NS1 induced cellular senescence in Neuro2a cells.

\section{Discussion}

The multifunctional NS1 protein of influenza A virus plays a pivotal role in viral infection by modulating many virus and host-cell processes. Our previous study has shown that NS1 protein of the novel influenza A virus H7N9 induces p53-mediated apoptosis of human A549 alveolar basal epithelial cells. Since the novel H7N9 influenza A virus can be detected in brain tissues in infected mice [34], we next examined whether H7N9/NS1 could induce the same apoptosis effect in Neuro2a cells as A549 cells. We observed significant cellular cytotoxicity and eventual cell death, but failed to detect obvious apoptosis in H7N9/ NS1-transfected Neuro2a cells. This is not particularly surprising given the fact that the biological activities of NS1 are likely to be stain and/or cell type specific [26]. Numerous studies have shown that several cell types including neurons infected by influenza viruses are capable of producing reactive oxygen and nitrogen species, including NO, which is served as a host response modulator during influenza virus infection [19]. NO can directly cause the protein nitrosylation, inhibit protein activities, and induce cell death [35]. In many types of cells exposure to excessive NO, through external addition or internally catalyzed by iNOS can cause DNA damage foci, lead to cellular senescence [24]. In a previous study, researchers demonstrated that NOS inhibitor, L-NMMA treatment resulted in significant improved survival rate after infection with a mouse-adapted H2N2 virus [16]. In another study, iNOS1- mice have shown to have reduced morbidity and reduced mortality to high pathogenic influenza virus. Besides, L-NMMA-treated mice infected with 1918 virus have been found to exhibit delayed weight loss and death compared to untreated mice [25]. NS1 has been reported as a key viral factor involved in virus triggered iNOS expression during influenza virus infection [27]. In the present study, the level of NO production and iNOS expression have been found to be positively correlated with NS1 expression level in Neuro2a cells and primary cultured mouse cortical neurons. Since the presence of continuous excessive NO can cause cell growth arrest, we generated a Neuro2a cell line that constitutively expresses H7N9/NS1 (NS1 O.E) to examine the effect of NS1 on cell proliferation. NS1 O.E cells can grow in the medium containing $10 \mathrm{mM} \mathrm{SMT}$, but exhibit a reduced proliferation rate compared to WT cells. However, NS1 O.E cells kept in the absence of SMT cannot survive for 7 days, and displayed a number of characteristic features, such as enlarged morphology, increased SA- $\beta$ gal activity, typical punctuate structures of pHP1 $\gamma$ in the nuclei and enhanced expression of IL-6 and IL-8 at day 3, which strongly suggesting that cells undergo senescence. In summary, 
our data suggest that sustained and elevated NO generated by iNOS could drive cell fate decision towards senescence.

Cell fate decisions in response to extracellular factors, including pathogenic invaders are commonly modulated by intracellular signal transduction cascades. p38 MAPK pathway has been shown to be critically involved in the control of cellular stress responses such as cell death, survival and senescence [36]. It has been reported that influenza A virus activate p38 MAPK in a Toll-like receptor4 -mediated manner during the early stage of infection, and utilize p38 to enter cell and replicate [37]. A recently study revealed that more than $90 \%$ of all virus-induced genes were either partially or fully dependent on p38 signaling in H5N1 and H7N7 influenza virus infected mice [38]. p38 MAPK has been reported to be involved in various steps in the influenza A virus life cycle. Influenza A virus activated-p38 MAPK has been found to contribute to both virally induced apoptosis and virus replication in human neuroblastoma cells SH-SY5Y [39]. In the present work, p38 MAPK in NS1 O.E Neuro2a cells was significantly activated relative to Neuro2a WT cells. The expression of phosphorylated p38 MAPK in NS1 O.E (+SMT) was much lower than that in NS1 O.E (-SMT), giving a clue that suppression of NO signaling pathway might inhibit the activation of p38MAPK. Although the specific mechanism remains unclear, it cannot be ruled out the influence of enhanced expression of iNOS and excessive NO on p38 MAPK pathway in NS1 O.E (-SMT), since an early study revealed that p38 MAPK is significantly activated in the presence of SNP, a NO donor, in human SH-SY5Y neuroblastoma cells and primary cultures of cortical neurons [32]. On the other hand, p38 inhibitor FHPI reduced the expression of iNOS in a dose-dependent manner, indicating that blockade of p38 MAPK pathway inhibits iNOS expression, reduces the production of NO and suppresses cellular senescence in Neuro2a cells. However, the complicated mechanism of how p38 MAPK pathway regulates iNOS expression in NS1expressed Neuro2a cell has yet to be expored. Nevertheless, our findings suggest that activation of p38 MAPK is involved in NS1 induced cellular senescence in Neuro2a cells.

Cellular senescence has been recognized as an alternate cell fate in the absence of apoptosis. It is not only restricted to the reduced replicative ability, but associated with changes in cellular metabolism, epigenetic regulation and gene expression [40]. In brain, neurons senescence might be implicated with several age-related neurodegenerative diseases [41]. A recent study suggested that cellular senescence can contribute to increased viral replication. Senescence-associated changes lead to an increased susceptibility of senescent cells to viral infection [42]. From this perspective, H7N9/NS1-induced senescence might be beneficial to virus replication and virus pathogenicity.

In conclusion, our present work shows that NS1 protein derived from a novel avian influenza virus induces NO-mediated cellular senescence in Neuro2a cells. As far as we know, this is the first time for NS1 protein of influenza virus to be associated with cellular senescence, which might extend our understanding on the contribution of NS1 to influenza virus pathogenicity.

\section{Acknowledgements}

This work was supported by the National Natural Science Foundation of China (grant number: 81471622) and China Postdoctoral Science Foundation Funded Project (Project No.: 2016M602498).

\section{Disclosure Statement}

The authors declare that they have no Disclosure Statement. 


\section{Cellular Physiology Cell Physiol Biochem 2017;43:1369-1380

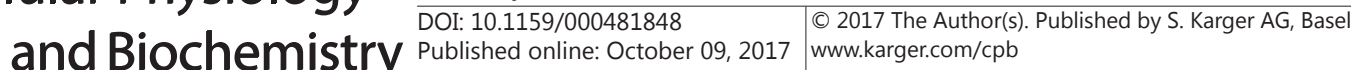

Yan et al.: H7N9 NS1 Protein Induces Cellular Senescence

\section{References}

1 World Health Organization. Antigenic and genetic characteristics of zoonotic influenza viruses and development of candidate vaccine viruses for pandemic preparedness, March 2017 Geneva, Switzerland: World Health Organization; 2017. http://www.who.int/influenza/vaccines/virus/201703_zoonotic_ vaccinevirusupdate.pdf?ua=1.

- Wang C, Yu H, Horby PW, Cao B, Yang S, Gao H, Li H, Tsang TK, Liao Q, Gao Z, Ip DK, Jia H, Jiang H, Liu B, Ni MY, Dai X, Liu F, Van Kinh N, Liem NT, Hien TT, Li Y, Yang J, Wu JT, Zheng Y, Leung GM, Farrar JJ, Cowling BJ, Uyeki TM, Li L: Comparison of Patients Hospitalized With Influenza A Subtypes H7N9, H5N1, and 2009 Pandemic H1N1 Clin Infect Dis 2014;58:1095-1103.

- 3 Deresiewicz RL, Thaler SJ, Hsu L, Zamani AA: Clinical and neuroradiographic manifestations of eastern equine encephalitis. N Engl J Med 1997;336:1867-1874.

-4 Glaser CA, Winter K,DuBray K, Harriman K, Uyeki TM, Sejvar J, Gilliam S, Louie JK: A population-based study of neurologic manifestations of severe influenza A (H1N1)pdm09 in California. Clin Infect Dis 2012;55:514-520.

5 Jang H, Boltz D, Sturm-Ramirez K, Shepherd KR, Jiao Y, Webster R, Smeyne RJ: Highly pathogenic H5N1 influenza virus can enter the central nervous system and induce neuroinflammation and neurodegeneration. Proc Natl Acad Sci U S A 2009;106:14063-14068.

6 Park CH, Ishinaka M, Takada A, Kida H, Kimura T, Ochiai K, Umemura T: The invasion routes of neurovirulent A/Hong Kong/483/ 97 (H5N1) influenza virus into the central nervous system after respiratory infection in mice. Arch Viro 2002;147:1425-1436.

7 Belser JA, Lu X, Maines TR, Smith C, Li Y, Donis RO, Katz JM, Tumpey TM: Pathogenesis of avian influenza (H7) virus infection in mice and ferrets: enhanced virulence of Eurasian H7N7 viruses isolated from humans. J Virol 2007;81:11139-11147.

8 de Wit E, Munster VJ, Spronken MI, Bestebroer TM, Baas C, Beyer WE, Rimmelzwaan GF, Osterhaus AD, Fouchier RA: Protection of mice against lethal infection with highly pathogenic H7N7 influenza A virus by using a recombinant low-pathogenicity vaccine strain. J Virol 2005;79:12401-12407.

-9 Kuiken T, Taubenberger JK: Pathology of human influenza revisited. Vaccine 2008;26:D59-D66.

10 Wiley CA, Bhardwaj N, Ross TM, Bissel SJ: Emerging Infections of CNS: Avian Influenza A Virus, Rift Valley Fever Virus and Human Parechovirus. Brain Pathol 2015;25:634-650.

11 Wang G, Li R, Jiang Z, Gu L, Chen Y, Dai J, Li K: Influenza Virus Induces Inflammatory Response in Mouse Primary Cortical Neurons with Limited Viral Replication. Biomed Res Int 2016:8076989.

12 Heneka MT, Feinstein DL: Expression and function of inducible nitric oxide synthase in neurons. J Neuroimmunol 2001;114:8-18.

13 Ricciardolo FL, Di Stefano A, Sabatini F, Folkerts G: Reactive nitrogen species in the respiratory tract. Eur J Pharmacol 2006;533:240-252.

14 Huang R, Zhang L, Gu Q, Zhou YH, Hao Y, Zhang K, Liu Y, Dong D, Wang S, Huang Z, Lu S, Wu C: Profiles of acute cytokine and antibody responses in patients infected with avian influenza A H7N9 PLoS One 2014;9:e101788.

15 Watanabe C, Kawashima H, Takekuma K, Hoshika A, Watanabe Y: Increased nitric oxide production and GFAP expression in the brains of influenza A/NWS virus infected mice. Neurochem Res 2008;33:10171023.

-16 Akaike T, Noguchi Y, Ijiri S, Setoguchi K, Suga M, Zheng YM, Dietzschold B, Maeda H: Pathogenesis of influenza virus induced pneumonia: involvement of both nitric oxide and oxygen radicals. Proc Natl Acad Sci U S A 1996;93:2448-2453.

-17 Snelgrove RJ, Edwards L, Rae AJ, Hussell T: An absence of reactive oxygen species improves the resolution of lung influenza infection. Eur J Immunol 2006;36:1364-1373.

18 MacMicking J, Xie QW, Nathan C: Nitric oxide and macrophage function. Annu Rev Immunol 1997;15:323350.

19 Akaike T, Maeda H: Nitric oxide and virus infection. Immunology 2000;101:300-308.

20 Heales SJ, Bolanos JP, Stewart VC, Brookes PS, Land JM, Clark JB: Nitric oxide, mitochondria and neurological disease. Biochim Biophys Acta 1999;1410:215-228.

-21 Chung KC, Park JH, Kim CH, Ahn YS: Tumor necrosis factor-alpha and phorbol 12-myristate 13-acetate differentially modulate cytotoxic effect of nitric oxide generated by serum deprivation in neuronal PC12 cells. J Neurochem 1999;72:1482-1488. 


\section{Cellular Physiology Cell Physiol Biochem 2017;43:1369-1380 \begin{tabular}{l|l|l} 
DOI: 10.1159/000481848 & $\begin{array}{l}\text { O 2017 The Author(s). Published by S. Karger AG, Basel } \\
\text { www.karger.com/cpb }\end{array}$
\end{tabular}}

Yan et al.: H7N9 NS1 Protein Induces Cellular Senescence

22 Heneka MT, Klockgether T, Feinstein DL: Peroxisome proliferator-activated receptor-gamma ligands reduce neuronal inducible nitric oxide synthase expression and cell death in vivo. J Neurosci 2000;20:6862-6867.

-23 Bonfoco E, Krainc D, Ankarcrona M, Nicotera P, Lipton SA: Apoptosis and necrosis: two distinct events induced respectively, by mild and intense insults with $\mathrm{N}$-methyl-D-aspartate or nitric oxide/superoxide in cortical cell cultures. Proc Natl Acad Sci U S A 1995;92:7162-7166.

24 Bagheri M, Nair RR, Singh KK, Saini DK: ATM-ROS-iNOS axis regulates nitric oxide mediated cellular senescence. Biochim Biophys Acta 2017;1864:177-190.

25 Perrone LA, Belser JA, Wasford DA, Katz JM, Tumpey TM: Inducible nitric oxide contributes to viral pathogenesis following highly pathogenic influenza virus infection in mice. J Infect Dis 2013;207:15761584.

26 Hale BG, Randall RE, Ortı'n J, Jackson D: The multifunctional NS1 protein of influenza A viruses. J Gen Virol 2008;89:2359-2376.

27 Li W, Yang F, Liu Y, Gong R, Liu L, Feng Y, Hu P, Sun W, Hao Q, Kang L, Wu J, Zhu Y: Negative feedback regulation of IL-32 production by iNOS activation in response to dsRNA or influenza virus infection. Eur J Immunol 2009;39:1019-1024.

28 Yan Y, Du Y, Deng Y, Wang G, Li R, Li K: The novel H7N9 influenza A virus NS1 induces p53-mediated apoptosis of A549 cells. Cell Physiol Biochem 2016;38:1447-1458.

29 Zhang H, Li W, Wang G, Su Y, Zhang C, Chen X, Xu Y, Li K: The distinct binding properties between avian/ human influenza A virus NS1 and postsynaptic density protein-95 (PSD-95), and inhibition of nitric oxide production. Viro J 2011;8:298.

-30 Sulli G, Di Micco R d'Adda di Fagagna, F: Crosstalk between chromatin state and DNA damage response in cellular senescence and cancer. Nat Rev Cancer 2012;12:709-720.

31 Coppe JP, Patil CK, Rodier F, Sun Y, Munoz DP, Goldstein J, Nelson PS, Desprez PY, Campisi J: Senescenceassociated secretory phenotypes reveal cell-nonautonomous functions of oncogenic RAS and the p53 tumor suppressor. PLoS Biol 2008;6:2853-2868.

-32 Ghatan S, Larner S, Kinoshita Y, Hetman M, Patel L, Xia Z, Youle RJ, Morrison RS: p38 MAP kinase mediates bax translocation in nitric oxide-induced apoptosis in neurons. J Cell Biol 2000;150:335-347.

33 Da Silva J, Pierrat B, Mary JL, Lesslauer W: Blockage of p38 mitogen-activated protein kinase pathway inhibits inducible nitric-oxide synthase expression in mouse astrocytes. J Biol Chem 1997;272:2837328380.

34 Bao L, Xu L, Zhu H, Deng W, Chen T Lv Q Li F, Yuan J, Xu Y, Huang L, Li Y, Liu J, Yao Y, Yu P, Chen H, Qin C: Transmission of H7N9 influenza virus in mice by different infective routes. Viro J 2014;11:185.

35 Hess DT, Matsumoto A, Nudelman R, Stamler JS: S-nitrosylation: spectrum and specificity. Nat Cell Biol 2001;3:46-49.

-36 Maruyama J, Naguro I, Takeda K, Ichijo H: Stress-activated MAP kinase cascades in cellular senescence. Curr Med Chem 2009;16:1229-1235.

37 Marchant D, Singhera GK, Utokaparch S, Hackett TL, Boyd JH, Luo Z, Si X, Dorscheid DR, McManus BM, Hegele RG: Toll-like receptor 4-mediated activation of p38 mitogen-activated protein kinase is a determinant of respiratory virus entry and tropism. J Virol 2010;84:11359-11373.

-38 Borgeling Y, Schmolke M, Viemann D, Nordhoff C, Roth J, Ludwig S: Inhibition of p38 Mitogen-activated Protein Kinase Impairs Influenza Virus-induced Primary and Secondary Host Gene Responses and Protects Mice from Lethal H5N1 Infection. J Biol Chem 2014;289:13-27.

-39 Nencioni L, De Chiara G, Sgarbanti R, Amatore D, Aquilano K, Marcocci ME, Serafino A, Torcia M, Cozzolino F, Ciriolo MR, Garaci E, Palamara AT: Bcl-2 expression and p38MAPK activity in cells infected with influenza A virus: impact on virally induced apoptosis and viral replication. J Biol Chem 2009;284:16004-16015.

40 Bree RT, Stenson-Cox C, Grealy M, Byrnes L, Gorman AM, Samali A: Cellular longevity: role of apoptosis and replicative senescence. Biogerontology 2002;3:195-206.

41 Tan FC, Hutchison ER, Eitan E, Mattson MP: Are there roles for brain cell senescence in aging and neurodegenerative disorders? Biogerontoogy 2014;15:643-660.

-42 Kim JA, Seong RK, Shin OS: Enhanced viral replication by cellular replicative senescence. Immune Netw 2016;16:286-295. 